\title{
ENTRE GENEALOGÍAS FAMILIARES Y GENEALOGÍAS POLÍTICAS: JÓVENES EN UN PROCESO DE COMUNALIZACIÓN MAPUCHE EN ARGENTINA
}

Laura Kropff

\section{Introducción}

Este artículo se enmarca en un objetivo general que se orienta a analizar cómo interpelaciones y articulaciones de agencia social que ponen en juego clivajes de edad y etnicidad intervienen en procesos de configuración de subjetividades que diversifican trayectorias políticas en procesos de organización mapuche en la provincia de Río Negro, Argentina. Tanto edad como etnicidad constituyen clivajes, es decir líneas que estructuran la organización social de identidades, subjetividades y agencias estableciendo dinámicas de agregación/desagregación (Briones \& Siffredi 1989). Se trata de clivajes que operan entramados (entre sí y con otros clivajes como clase, género, etc.) habilitando prácticas, estilos, performances y discursos en torno a modelos de subjetividad que se impregnan de valores morales. El concepto de clivaje implica concebir que las diferencias entre los grupos no son absolutas sino relativas, relacionales y contextuales. El análisis de edad y etnicidad en particular tiene que ver con el uso de categorías sociales que ponen estos clivajes explícitamente en juego en los procesos de organización mapuche.

Las últimas dos décadas del siglo XX vieron surgir un movimiento político supra-comunitario mapuche en las provincias del norte de la Patagonia. Como consecuencia de este movimiento, en la primera década del siglo XXI surgieron agrupamientos independientes de autodenominados “jóvenes" en diferentes ciudades de la provincia de Río Negro. Desde 2001 vengo siguiendo el devenir de algunos de estos grupos que desarrollan su actividad política, fundamentalmente, en ámbitos urbanos (Kropff 2004). El acompañamiento de sus procesos de organización me llevó a analizar su articulación con organizaciones de base rural desde 2008 (Kropff 2011). En 
continuidad con esa trayectoria, actualmente realizo trabajo de campo en contextos rurales y urbanos del oeste de la provincia de Río Negro.

En este artículo propongo una aproximación a los modos en que la articulación generacional de los "jóvenes mapuche" en el presente produce sentido sobre - y es definida por-tanto la genealogía política del movimiento de fines del siglo XX como la genealogía familiar y comunitaria. El análisis parte de pensar la interrelación entre las dinámicas parentales y los procesos históri$\cos$ y, en función de eso, me centro en el caso de la comunidad Newen Nuke Mapu ubicada en la cuenca del arroyo Comallo, en Río Negro. Presentaré, en primer lugar, los antecedentes que permiten enmarcar el caso en el proceso histórico de incorporación de los mapuche al estado nacional argentino. En segundo lugar, indagaré en el modo en que se trazaron genealogías en la reunión de constitución formal de la comunidad, ocurrida en 2011, para delinear la forma en que el clivaje etario opera en el proceso de comunalización. En tercer lugar, revisaré los modos en que los "jóvenes" que hoy integran la comunidad se posicionan con respecto a la genealogía familiar y comunitaria, para luego analizar la manera en que esa articulación se entrama con la genealogía política. Finalmente, compararé las interpelaciones en términos etarios que se dan en contextos de activismo y en contextos comunitarios y familiares, rastreando las experiencias de colectividad y articulaciones etarias que se producen en el proceso de re-construcción comunitaria. Esto implica analizar la reelaboración política generacional que los "jóvenes" realizan de los discursos organizacionales mapuche a partir de su puesta en práctica en el proceso de articulación comunitaria.

\section{Antecedentes}

El Pueblo Mapuche se define como un pueblo pre-existente a los estados nacionales de Chile y Argentina. Las organizaciones supra-comunitarias delimitan su territorio ancestral en términos geopolíticos, ubicándolo al sur del río Bío-Bío en Chile y en la Patagonia Argentina al sur de las provincias de La Pampa y Buenos Aires (Coordinación de Organizaciones Mapuche Taiñ Kiñe Getuam 1995).

La matriz estado-nación-territorio se fue consolidando en la Argentina hacia fines del siglo XIX, cuando se ocuparon militarmente territorios en los cuales todavía había pueblos indígenas autónomos. El territorio patagónico fue apropiado violentamente por dos estados nacionales que desarrollaron campañas militares paralelas: Chile y Argentina. En Argentina, la conquista militar que concluyó formalmente en 1885 implicó la ruptura de las formas 
autónomas de organización mapuche y fue la base de una política de usurpación y redistribución de tierras y personas. En ese sentido, se convirtió en un evento fundamental que asignó características particulares al proceso de territorialización (Pacheco de Oliveira 1998) de colectivos marcados como indígenas en la Patagonia.

La persecución y apresamiento en campos de concentración implicó la disposición arbitraria de los indígenas como mano de obra esclava para polos emergentes del norte del país desmembrando comunidades y familias. Fueron incorporados como sirvientes de la alta sociedad y como combatientes en las fuerzas armadas separando mujeres, hombres y niños (Mases 2002; Lenton 2005; Escolar 2008). Sin embargo, desde estos mismos campos de concentración se negociaron, en condiciones de franca asimetría, algunas entregas puntuales de tierra a figuras reconocidas por el estado. Estas tierras se ubicaban en zonas consideradas poco productivas (Briones y Delrio 2002; Delrio 2005). Quienes no consiguieron concretar la negociación, acabaron buscando la supervivencia en tierras fiscales sin título de propiedad.

Paralelamente a la conquista militar y a la lenta reubicación de la población indígena, se consolidó un proyecto nacional que, basado en la doctrina de "civilización o barbarie", fomentó la inmigración europea para "mejorar la sangre" de los criollos (Svampa 1994). Este planteo ideológico favoreció la negación de la presencia indígena en general y el desarrollo de estrategias de des-marcación de la identidad por parte de los afectados. En ese marco, la categoría "indio" incluyó características racializadoras y etnicizadoras, así como implicaciones en términos de falta de adecuación a la lógica productiva (Radovich y Balazote 1995). Estos argumentos, que ya habían sido fundamento para la ocupación militar de fines del siglo XIX, fueron retomados para justificar desalojos y persecuciones entrado el siglo XX (Pérez 2009).

El estado argentino comenzó a reconocer la presencia de Pueblos Indígenas en su territorio recién en la década de 1990. Hasta la reforma constitucional de 1994, que reconoce la preexistencia étnica y cultural de los Pueblos Indígenas (art. 75, inc. 17), la única mención a los mismos en la carta magna recomendaba mantener un trato pacífico y promover su conversión al catolicismo. Una vez reconocidos, el estado se vio en la obligación de saber cuántos eran y dónde estaban ubicados los indígenas. Por ello, en el Censo Nacional de Población y Vivienda de 2001, se incluyó por primera vez una pregunta referente a la identidad indígena de la población. El criterio que debía utilizarse para medir era el de autoreconocimiento y no criterios biologicistas o culturalistas. Por lo tanto, teniendo en cuenta que la construcción de identidades es consecuencia de un proceso relacional y 
que, en este caso, se trataba de una relación históricamente asimétrica, el censo propuso una situación compleja. El estado argentino reconoció por primera vez tener población indígena después de más de cien años de una política negadora y derogatoria. Ante esto, varias organizaciones mapuche advirtieron que el número resultante no daría cuenta de la cantidad de indígenas sino de los efectos de las políticas extincionistas y asimilacionistas del propio estado nacional (Organización Mapuche Newentuayiñ y Pu weche fiske menuko 2001).

Entre los años 2004 y 2005 se realizó la Encuesta Complementaria de Pueblos Indígenas. Como resultado, 113.680 personas se reconocieron descendientes o pertenecientes al Pueblo Mapuche. El 71,6\% de las personas que se reconocieron como indígenas se incluían en sectores urbanos. En el 8,2\% de los hogares censados en 2001 en la provincia de Río Negro al menos un integrante se reconoció descendiente o perteneciente a algún Pueblo Indígena. ${ }^{1}$ Sin embargo, considerando las limitaciones del proceso de construcción de estos datos, sólo podemos tomarlos como una referencia que no permite elaborar conclusiones demográficas certeras. Lo que sí puede afirmarse es que las políticas estatales y las acciones de privados han promovido la expulsión de los mapuche de las áreas rurales para generar latifundios. Como efecto de este proceso, la población mapuche hoy se concentra mayoritariamente en los barrios marginales de las ciudades que se emplazan en lo que fue territorio autónomo hasta fines del siglo XIX.

En cuanto a las áreas rurales, encontramos que las políticas de radicación redundaron en la configuración de algunos colectivos indígenas que lograron negociar tierras tempranamente, así como otros que nunca fueron reconocidos como tales por el estado. En algunos casos fueron reconocidos como indígenas pero no como comunidad (Cañuqueo, Kropff y Pérez 2015). En ese sentido, "comunidad" es una categoría de uso social con implicaciones políticas y jurídicas específicas. Del mismo modo, en este contexto, la noción mapuche de lof constituye una categoría de uso social y político especialmente visibilizada en situaciones en las que hay conflicto territorial con privados.

La categoría lof implica, en términos generales, una articulación de procesos de territorialización y de tramas de relacionalidad descritas a partir del lenguaje del parentesco. El lof se inscribe tanto en espacios ceremoniales como en clave territorial (Cañumil y Ramos 2012). Se trata de una categoría polisémica en la que confluyen significados que provienen del ámbito de lo ceremonial con otros del ámbito de lo político y hasta de lo jurídico (Cañuqueo 2011). Su definición siempre debe ser entendida en función del contexto en el que se enuncia. En algunos contextos los términos lof y "comunidad" son sinónimos mientras que, en otros, no es así. 
En el caso que analizo aquí tenemos un colectivo mapuche que decide articularse como "comunidad" incluyendo pobladores de distintos parajes y un lof. El término lof identifica, en esta zona, a grupos parentales que se encuentran en procesos de reivindicación territorial que se dirimen tanto en la arena política como en la jurídica. Así, el conflicto territorial es indexicalizado por el uso del término lof.

\section{La comunidad Newen Ñuke Mapu}

La Newen Nuke Mapu [Fuerza de la Madre Tierra] se constituyó formalmente como comunidad mapuche en agosto de 2011. Nuclea a más de cuarenta familias de seis parajes rurales de la cuenca del arroyo Comallo en Río Negro. El área se incluye en la región denominada Línea Sur, destino histórico de radicación de familias mapuche luego de la conquista militar del territorio a fines del siglo XIX. En su mayoría, los pobladores no cuentan con títulos de propiedad de la tierra que ocupan, sino con permisos de ocupación precaria. Los integrantes de la Newen Nuke Mapu están radicados en estos parajes y también en las localidades urbanas de Comallo y Bariloche.

La constitución formal de la comunidad fue efecto de procesos colectivos de larga duración que cristalizaron en 2011 debido a una circunstancia de orden natural que afectó profundamente la vida social. En junio de ese año entró en erupción el cordón Caulle-Puyehue, situado al oeste de la cordillera de los Andes. La orientación de los vientos Oeste/Este produjo una importante lluvia de ceniza que llegó hasta el Océano Atlántico. Entre otros efectos, la economía de la Línea Sur, basada en la cría de ganado ovino y caprino, se vio profundamente alterada. La coyuntura de emergencia ganadera hizo que se multiplicaran las reuniones entre pobladores damnificados de las zonas rurales. En este contexto de emergencia, se desarrollaron diferentes estrategias de articulación entre pobladores y referentes gubernamentales encaminadas a obtener respuestas efectivas.

Una de las estrategias llevadas adelante fue la conformación de la comunidad de acuerdo a las pautas requeridas por los organismos que aplican políticas para los Pueblos Indígenas. La figura de la "comunidad indígena" que los organismos reconocen combina aspectos de tipo jurídico con ciertas nociones estandarizadas de lo que se entiende por tradición (Cañuqueo 2010). Para los pobladores de los parajes, la función que debe cumplir la comunidad reconocida jurídicamente es organizar demandas y gestionar soluciones que no se pueden viabilizar a través de otras instan- 
cias de organización colectiva como las cooperativas de productores, las comisiones de fomento, etc.

La reunión en la que se decidió conformar la comunidad se realizó en la casa de un vecino y hubo presencia de pobladores de los seis parajes, además de gente de Bariloche y de Comallo y de algunos miembros de organizaciones urbanas, entre ellos varios de los jóvenes con los que yo venía trabajando. Uno de los momentos más emocionantes de esa reunión fue la mención de una carta presentada a la Comisión Honoraria de Reducciones de Indios en el año 1941. La carta, avalada por 54 firmas, solicitaba que el área comprendida por cinco de los parajes que actualmente integran la comunidad fuera reconocida como Reducción de Indígenas argentinos. La respuesta recibida en su momento negó esa solicitud con argumentos racistas y derogatorios, dando lugar a políticas atomizadoras que derivaron en la tramitación individual de las certificaciones de tenencia precaria y los títulos de propiedad (Cañuqueo, Kropff y Pérez 2007 y 2008). Sin embargo, su valor en el contexto de la reunión tenía que ver con que constituía un antecedente de demanda colectiva indígena en la zona. ${ }^{2}$ Luego de comentar el contenido de la demanda, se leyó en voz alta la nómina de los que la firmaban. Cada vez que se nombraba un firmante alguno de los presentes lo señalaba como pariente suyo. "Es mi señor padre", dijo un vecino, y otros fueron reconociendo abuelos, tíos, tías etc. Ése fue el punto de partida para el análisis de la situación actual que incluyó distintos tópicos como la tierra, la emergencia y la organización comunitaria.

\section{Genealogías, comunalización y espacialización}

La referencia a la carta, en la instancia de conformación formal de la comunidad, la convirtió en un símbolo potente que operó sobre varias dimensiones fundamentales del proceso de comunalización. Según James Brow (1990) la comunalización es un proceso continuo que promueve un sentido de pertenencia colectivo que combina componentes afectivos y cognitivos. En este proceso se van sedimentando sentidos, de modo tal que ciertas relaciones sociales que están cultural e históricamente determinadas se presentan como si fueran naturales. A esto llama Brow "primordialización", estableciendo una relación con el concepto de doxa de Pierre Bourdieu que implica la percepción del mundo socialmente construido como si fuera un orden auto-evidente y natural que se da por sentado. En este sentido, la primordialización es exitosa cuando logra naturalizar la arbitrariedad de las relaciones comunales. Sin embargo, el campo de la doxa siempre co-existe con un campo de discusión. Así, la lectura de la carta operó primordializando 
al colectivo indígena que se estaba constituyendo formalmente como comunidad. Se trata de un colectivo que no fue reconocido históricamente como tal por el estado, lo que redunda en que la noción misma de comunidad se encuentre en un campo de discusión y no incluida en la doxa.

Ahora bien, la primordialización implica una serie de operaciones que la lectura de la carta permitió realizar. Por un lado, la lectura proyectó la noción de comunidad al pasado, poniendo en cuestión las interpelaciones atomizadoras que imperaron desde entonces, e instaurando una tradición de organización y demanda colectiva de derechos, fundamentalmente del derecho a la tierra. Por otro lado, contribuyó a legitimar la identidad indígena de los allí presentes porque, si bien el estado siempre dialogó con los pobladores de los parajes interpelándolos con categorías etnicizadas (indígenas, aborígenes, mapuche, etc.), la etnicidad no está garantizada para quienes viven en espacios urbanos, como veremos más adelante. La primordialización de la comunidad y de la etnicidad se garantizó a través del lenguaje del parentesco por su apelación a la consanguinidad y por el trazado de genealogías. La apelación a la consanguinidad permitió naturalizar, tanto la identidad étnica de todos los allí presentes (incluyendo a los urbanos) como la continuidad histórica de la comunidad y, por ende, de su lucha por la tierra.

Además de la apelación a los lazos de sangre, el lenguaje del parentesco permite naturalizar el trazado de genealogías que colocan a los jóvenes como herederos de los mayores. ${ }^{3}$ Esta expresión de la dimensión etaria en el marco del proceso de comunalización es la que me interesa profundizar aquí. La inscripción de una genealogía conlleva supuestos implícitos acerca de la herencia la transmisión de derechos y obligaciones, la memoria y la proyección hacia el futuro, entre otros (Irvine 1978). En este caso, se trata de una inscripción centrada en la relación entre la comunidad y el espacio que habita.

Ya en el proceso de organización supra-comunitaria que ganó visibilidad en las últimas décadas del siglo XX, las categorías a partir de las cuales el espacio es apropiado socialmente como lugar (Gupta y Ferguson 1992) devinieron objeto de reflexión política pero también biográfica y genealógica. Así, la definición de Wajmapu [territorio mapuche] opera en el ámbito del movimiento mapuche contemporáneo indexicalizando el proceso histórico de territorialización (Pacheco de Oliveira 1998) basado en la usurpación y despojo llevado a cabo por los estados nacionales de Chile y Argentina desde fines del siglo XIX.

Pero hay otras categorías espacializadoras que intervienen en este proceso, siendo una de las marcaciones centrales la dualidad campo/ciudad en tanto construcción asimétrica de lugar que se instauró en el proceso de consolidación y expansión del capitalismo y que devino lógica explicativa de 
lo social (Williams 2001 [1973]). Esta construcción naturaliza las relaciones sociales de producción impuestas, espacializando subjetividades también asimétricas basadas en la oposición fundante de la modernidad: civilizado/ salvaje (Svampa 1994). A la vez, se trata de un esquema que espacializa el tiempo. Por un lado, coloca el pasado en el espacio rural y el presente/ futuro en el espacio urbano y, por otro lado, define al campo como el lugar de lo estático y a la ciudad como el lugar del cambio. En términos étnicos, los mapuche quedan circunscriptos al campo y, en esa operación, también quedan circunscriptos al pasado (Szulc 2004). En términos etarios, los jóvenes, cuya condición naturalmente transicional les otorga el futuro (Chaves 2010), quedan circunscriptos a las ciudades. El ámbito rural es, por lo tanto, un ámbito donde lo juvenil se vuelve impensable (González Cangas 2003). De allí que la subjetividad definida como "joven mapuche rural" devenga desafiante (Kropff 2011).

\section{Algunas precisiones conceptuales sobre la edad}

Desde la perspectiva teórica que adopto en este análisis, "juventud" no es un concepto sino una categoría de uso que opera en una estructura de alteridades etarias. Del mismo modo, "mapuche" no es un concepto sino una categoría de uso que opera en la arena definida por la construcción de aboriginalidad (Beckett 1988; Briones 1998).

En 2010, Valentina Stella y yo realizamos un relevamiento sobre los estudios que abordan la relación entre juventud y etnicidad en Latinoamérica. De ese relevamiento surgió un panorama de las perspectivas teóricas presentes en el corpus bibliográfico. En primer lugar, encontramos perspectivas que toman la condición etaria y la identidad étnica como punto de partida para abordar, desde allí, problemas que afectan a los sujetos definidos previamente desde esas categorías. Un segundo conjunto de abordajes considera la juventud como dato biológico/cronológico y construye la etnicidad como problema. Finalmente, el último grupo de investigaciones se basa en la definición de la etnicidad como dato (fundado en contenidos culturales y/o en estadísticas nacionales/estatales) y problematiza la juventud. Sobre la base de la revisión de los aportes provenientes de estos tres enfoques, propusimos la problematización de edad y etnicidad como clivajes que definen la movilidad estructurada de subjetividades cuya trama debe leerse situada en cada contexto sociopolítico e histórico (Kropff y Stella 2010).

Ahora bien, mientras los estudios étnicos tienen una larga tradición en el análisis de la etnicidad en estos términos (textos clave son Barth 1969; 
Cardoso de Oliveira 1971), dando un debate temprano entre posturas substancialistas, formalistas, instrumentalistas y materialistas (ver recorrido del debate en Briones 1998 y Pacheco de Oliveira 1998) no son tantas las perspectivas que consideran la edad como clivaje.

Un aporte fundamental en esta línea es el de Deborah Durham (2000), quien propone pensar la categoría "juventud" como un índice [shifter], o sea como un tipo especial de deíctico que vincula al hablante con un contexto relacional o indexical (Silverstein 1976). Así, el uso de la categoría juventud construye un contexto conformado, en principio, por relaciones asimétricas (Pérez Ruiz 2008) expresadas en términos de alteridades etarias. Además, este movimiento conceptual nos lleva más allá de las relaciones sociales que se negocian en el momento, para orientar la atención hacia el paisaje social. No sólo se indexicaliza la trama de alteridades etarias sino también nociones de persona, valores morales y supuestos cronotopológicos sobre la relación entre pasado y futuro, entre otros aspectos de la vida social.

Siguiendo esta propuesta, resulta relevante preguntar qué condiciones habilitan, promueven y limitan el uso de la categoría "juventud" en diferentes contextos, y qué efectos genera este uso en términos tanto de interpelación como de articulación de agencia. En este sentido, los distintos contextos en los que la categoría "juventud" aparece asociada a "indígena" han llevado a los investigadores a reflexionar sobre la relación asimétrica entre las teorías de los distintos pueblos originarios en torno a la edad (que pueden o no incluir una categoría denominada "juventud") y la teoría occidental promovida por el avance de los estados nacionales y el capitalismo global sobre las comunidades (Pérez Ruiz 2008). Los estudios que trabajan en este campo ponen en evidencia (etnográfica) la arbitrariedad de la edad como dimensión estructurante de las prácticas sociales, así como los efectos situados e históricos de la asimetría entre las distintas teorías en relación a la configuración de subjetividades. De modo que, en el contexto que analizo aquí, no es únicamente el uso de la categoría "mapuche" el que indexicaliza el proceso de consolidación de la matriz estado-nación-territorio, sino que el uso de la categoría "joven" también está configurando ese paisaje social, haciendo énfasis en la consolidación de esta matriz en relación al capitalismo de la segunda mitad del siglo XX.

\section{Acerca de la polisemia de las categorías etarias}

Entonces, el capital simbólico de las categorías que definen "grados de edad" (Radcliffe-Brown 1929) se pone en juego en condiciones de asime- 
tría y opera naturalizando pautas de conducta y conjuntos de roles (Kertzer 1978) que difieren en distintos contextos. Recordemos que por "grados de edad" Radcliffe-Brown alude a "divisiones reconocidas de la vida de un individuo mientras pasa de la infancia a la vejez" (Radcliffe-Brown 1929:21). El concepto de "grado de edad" ilumina las interpelaciones que constituyen las arenas contextuales e históricas definidas por las diferentes categorías etarias que inscriben subjetividades (Müller-Dempf 1991).

Sin embargo, las categorías etarias puestas en uso no siempre hacen referencia a grados de edad. En el intento teórico por complementar el análisis de la categoría "juventud" con una perspectiva diacrónica que dé cuenta de los procesos históricos específicos en los que se pone en juego, Deborah Durham retoma el uso que Jean y John Comaroff hacen del concepto de "generación" para referirse a los desplazamientos en la experiencia que acaban creando cohortes con conciencia de edad [age-conscious cohorts], lo que los antropólogos clásicos llaman "grupos de edad". Los grupos de edad -originalmente registrados en las etnografías africanas de principios del siglo XX como instituciones sociales basadas en la solidaridad etaria que son transversales a las estructuras de linaje y que se conforman en instancias rituales (ver, entre otros, Evans-Pritchard 1987 [1940]) - se fundan en sensibilidades que están en íntima relación con experiencias sociales significativas compartidas y sancionadas públicamente, o sea, ritualizadas (Maybury-Lewis 1974 [1967]). Entendida como grupo de edad, la generación constituye una articulación de agencia altamente naturalizada que, lejos de ser universalizable, es permanentemente negociada y resignificada.

En tanto grupos de edad que atraviesan grados de edad, la dinámica de las generaciones produce sentido en torno al flujo de la experiencia social, otorgando interpretaciones que fijan coordenadas temporales para marcar continuidades y rupturas en el(los) sentido(s) de devenir a partir de la inscripción de las "experiencias originarias" - las primeras con las que una generación ingresa en la arena social como actor colectivo (Lewkowicz 2003) - como mojones en el flujo del tiempo.

Ahora bien, no en todos los casos el concepto antropológico de generación refiere a grupo de edad, sino que fluctúa en la ambivalencia entre esa definición basada en la experiencia social compartida (Radcliffe-Brown 1929) y la noción de cohorte genealógica demarcada por el nacimiento (Kertzer 1978; Müller-Dempf 1991). Esta ambivalencia permite explorar la confluencia entre parentesco, política y dinámicas históricas en los grupos humanos.

En nuestra sociedad, a diferencia de las comunidades africanas descritas en las etnografías clásicas, la estructura de grupos de edad no funciona 
como una institución específica, demarcada por ritualizaciones reconocidas y nombres propios. La generación se define, entonces, en la interfase entre la genealogía y los procesos de conformación de grupos de edad a partir de experiencias colectivas con diferentes grados de ritualización y reconocimiento público. Esto genera que el lenguaje del parentesco opere en dos direcciones. Por un lado, substancializando relaciones sociales (Alonso 1994) y, por otro, inscribiendo procesos sociales en las trayectorias genealógicas, historizándolas.

\section{Los “jóvenes mapuche" en la genealogía comunitaria}

Ahora, volviendo al caso analizado, las perspectivas de los autodenominados "jóvenes" que nacieron en las ciudades, cuyos padres migraron desde los parajes rurales, y que hoy buscan un vínculo con sus comunidades de origen, están, en general, atravesadas por experiencias personales en las que la autoadscripción como mapuche se produjo durante la adolescencia o con posterioridad. Esto se debe a que la movilización de las organizaciones mapuche recién consiguió instalar un lugar legítimo de identificación pública en la década de 1990 (Briones 2006; Radovich y Balazote 2000, entre otros). Hasta esa década, la experiencia de los jóvenes de los barrios urbanos estaba atravesada por la discriminación y la invisibilización, producto de la hegemonía asimilacionista (Kropff 2002).

Una de las jóvenes que hoy integra la comunidad Newen Nuke Mapu hizo referencia a su descubrimiento de sí misma en tanto mapuche a través de su inscripción ceremonial en una genealogía, cuando me narró su primera experiencia de participación en el kamarikun [ceremonia religiosa]:

Todos los que llegaban se presentaban. Y mi vieja también se presentó y dijo que ella tenía este apellido pero que su mamá tenía otro y que era de tal paraje y entonces ahí las abuelitas pararon las orejas. Y al otro día, cuando terminó toda la actividad del día la llamaron a mi mamá y a mi hermana, porque viste que ahí es con su hija mayor. Y le dijeron que mi abuela participaba en los kamarikun, la mamá de ella. Y eso fue otro gran momento porque vos decías... cada minuto que pasaba, cada cosa que hacías en la ceremonia, vos decías 'estoy haciendo lo que hacía mi abuela hace... no sé, cincuenta años atrás'. Y que lo hicieron sus abuelos también (...) A mi mamá le dijeron que ella era como de la casa también porque su mamá había ido ahí. Y que su mamá era una mujer muy respetada (...) Era como que empezamos a ver y decir... dar vuelta al rewe [lugar central del espacio ceremonial], hacer tayvl [canto sagrado] era hacer lo mismo que estaba haciendo tu abuela hace unos años atrás. Como que estás retomando 
todo (...) Como que hubo una palabra presente durante años y que después esa palabra se vuelve a decir en el mismo lugar y en el mismo espacio... con otras generaciones, se transmite (...) Era como que vos caminabas y tu abuela iba al lado. Y yo también decía que tenía la responsabilidad de hacer lo mejor posible las cosas porque mi abuela estaba... me lo exigía mi abuela... había hecho eso (...) Es como que es la memoria en la sangre, la llamaba uno de los chicos (...) Era como que ya lo habíamos vivido pero en realidad vos te ponías a pensar y en tu vida no lo habías vivido. Entonces eso él lo llamaba la memoria en la sangre (...) Era retomar el círculo. Es fuerte porque es encontrarte con vos mismo.

En este caso, el monitoreo colectivo que, quienes se definen como jóvenes, hacen de su experiencia, los lleva a objetivar las prácticas de transmisión de saberes e interpretaciones del pasado que los colocan en una línea genealógica y comunitaria mapuche. La transmisión, en el fragmento citado arriba, tiene que ver con prácticas ceremoniales religiosas que se desarrollan "en el mismo lugar". El espacio, devenido lugar por la apropiación social y la asignación de una identidad distintiva (Gupta y Ferguson 1992) y sedimentada a lo largo de la historia permite colocar las prácticas propias en un devenir en el cual tienen la propiedad performativa de activar la transmisión: "retomar el círculo" reforzando la cualidad distintiva de ese espacio/lugar.

Otro proceso de inscripción espacial de la genealogía comunitaria es el de reafirmación territorial. Para explicar esto, resulta particularmente significativo el caso del lof Mariano Epulef que integra la comunidad Newen Nuke Mapu. Uno de los firmantes de la carta de 1941, cuyo nombre se leyó en el encuentro de conformación de la comunidad, es Benito Linares, casado con una hija de Mariano Epulef. En la reunión había hijos y nietos de Linares que reconocieron explícitamente la genealogía. De hecho, el lof se define a sí mismo en función de una articulación genealógica en tanto se compone de tres generaciones de descendientes de Mariano Epulef.

El lof presentó, en el año 2004, un reclamo judicial exigiendo la restitución de 13.500 has de tierra que fueron compradas en forma fraudulenta por el terrateniente Ramón Fernández en la década de 1940 y que actualmente - año 2015 - se encuentran en poder de una sociedad anónima. Esta expropiación fraudulenta hecha en connivencia con los poderes de estado obligó a varios de los miembros del lof a migrar o a trabajar para el propio Fernández en el servicio doméstico o como peones rurales (Cañuqueo, Kropff y Pérez 2007). Actualmente, los integrantes del lof residen en el paraje Anecón Chico y en diferentes pueblos y ciudades de la provincia de Río Negro. En el lof confluyen trayectorias urbanizadas y trayectorias ruralizadas que 
configuran los posicionamientos etarios y las pertenencias étnicas de modos diferentes. ${ }^{4}$ Basándose en la producción de sentido sobre esta experiencia histórica, el lof reclama el reconocimiento de ocupación ancestral de un espacio territorial que nunca abandonó. Por ese motivo, define su demanda en términos de reafirmación y no de recuperación territorial.

El relato de los integrantes del lof se basa en un esquema de generaciones genealógicas atravesadas por diferentes experiencias sociales vinculadas a la relación con el estado y con los latifundistas y a la participación política en organizaciones campesinas y mapuche. El relato se origina con Mariano Epulef, que se radicó en el paraje Anecón Chico alrededor de 1890, en un período caracterizado por los desplazamientos de familias y comunidades producido por efecto de las campañas militares estatales de fines del siglo XIX y de las políticas genocidas que se instauraron con posterioridad. La generación de sus hijos realizó demandas al estado para lograr el reconocimiento legal de su radicación en la década de 1940. La generación de sus nietos vio avanzar los alambres de los latifundistas sobre los campos que tradicionalmente ocupaban las familias mapuche. El avance de los alambres se hizo en connivencia con los poderes del estado siendo, en muchos casos, los mismos latifundistas los que actuaban como funcionarios estatales. Una gran parte de los miembros de esa generación atravesó la experiencia de migrar a la ciudad y trabajar en oficios urbanos, como la construcción y el servicio doméstico. La toma de conciencia sobre estas condiciones estructurales e históricas de opresión fue uno de los factores que confluyeron en la conformación de organizaciones indígenas supra-comunitarias así como de cooperativas de productores, organizadas en torno a la condición campesina en la década de 1980.

Finalmente, los bisnietos de la generación de Epulef, criados en el campo y en la ciudad, atravesaron el período de revitalización de las prácticas tradicionales y de reivindicación identitaria de la década de 1990, que incluyó programas políticos basados en los conceptos de Territorio, Comunidad, Pueblo, Autonomía y Autodeterminación. Una generación cuya experiencia de socialización y formación política se dio en un contexto de continuidad democrática, de emergencia de movimientos sociales, etc. Son ellos los que impulsan la reafirmación territorial y, en este caso, su lof les traspasa el poder de tomar decisiones. En el contexto presente, conviven dos de esas generaciones, la que vivió el avance de los alambres y la que los desata para afirmarse en el Territorio. A ellas se suma la generación de los hijos de los más jóvenes que aún son niños. Todas están insertas en una trama social en la que la toma de decisiones y los lugares comunitarios se rigen por la jerarquía genealógica y las obligaciones de parentesco. 
Un día, cuando uno de los "jóvenes" del lof me comentaba un incidente en el que tuvo que confrontar a privados y policías que estaban arriando sus animales de uno de los cuadros que está en disputa, me dijo:

Yo he tomado cosas de mi tía, de mi tío, de mi otro tío, mucho más que de otras personas (...) Me di cuenta de que hay animales que son de la familia y es gracias a que lo empezó Epulef. No son los mismos animales pero él empezó, después la abuela acá, el abuelo... y continuaron otras generaciones y llega a nosotros. Nosotros, por ejemplo, no tuvimos necesidades como las que cuentan ellos. No vivimos tiempos difíciles. Y gracias a que ellos se han sacrificado nosotros tenemos esa posibilidad. Entonces es como querer devolver algo. No es sentirse en deuda sino que es devolver lo que nos dieron cuando éramos chicos (...) Y, entonces, en el momento que vienen a hacer el arreo, a mí me salió eso automático. Es como que se prendió el piloto automático de resistir y saber que podía hacerlo. (...) En ese momento sentí la excitación de la guerra. Fue algo más. Cuando eso lo recuerdo, lo vivo. Ahí nací. Ahí fue donde nací. (...) Yo me acuerdo siempre de que mi tía me decía: yo lo que quiero es que vos tengas valor. Lo más importante en la vida es el valor. Y yo le decía pucha tía, por qué no me decías que sea rico, que sea un ganador con las mujeres, por qué no me bendecís para que sea así [risas]. No, lo más importante, hijo, es el valor. Hay que ser valiente (...) En ese momento sentí el poder. Sé lo que es el poder. (...) A pesar de que éramos dos nomás contra todos ellos, había mucha más gente ahí con nosotros que nos dio fuerza.

El "nosotros" de este relato no es específicamente juvenil, sino un nosotros ubicado en una genealogía familiar. La posición etaria como categoría autoadscriptiva está operando en un proceso de comunalización e indexicalizando una estructura de alteridades que no tiene que ver con grados de edad (en tanto subjetividades inscriptas en la trama social en clave etaria), sino con alteridades etarias en una genealogía que está siendo narrada desde un marco discursivo familiar/comunitario.

\section{Los “jóvenes mapuche” en la genealogía de las organizacio- nes supra-comunitarias}

En el caso mapuche, la incorporación de los autodenominados "jóvenes" dentro del horizonte activista tuvo dos momentos significativos en términos de emergencia pública: la década de 1990 y la primera década del siglo XXI. En la década de 1990, las organizaciones de los Pueblos Indígenas lograron instalar públicamente el cuestionamiento a los planteos extincionistas y 
asimilacionistas que eran hegemónicos en Argentina. Sobre la base de ese cuestionamiento y de la revisión política de los principios fundantes del estado nacional, lograron el reconocimiento de algunas reivindicaciones. En ese contexto de demanda, uno de los reconocimientos más importantes fue la inclusión de un artículo en la Constitución Nacional reformada en 1994 (art. 75, inc. 17). Ese artículo reconoce la pre-existencia de los Pueblos Indígenas al estado nacional y, en consecuencia, una serie de derechos incluyendo la propiedad de las tierras que tradicionalmente ocupan.

Las organizaciones mapuche, cuyos referentes eran considerados "jóvenes" en ese momento, participaron activamente de ese movimiento y lograron construir, a pesar de sus múltiples diferencias internas, una agenda común en lo referente a los derechos territoriales de las comunidades y al respeto a la diferencia cultural (Radovich y Balazote 2000). La agenda incluye lo que se denomina como "recuperación cultural", que se centra en la recuperación de rituales, lenguaje y kimvn [conocimiento]. Esto se enmarca en un programa que implica un proceso de producción cultural orientado, por un lado, a la construcción de una idea de comunidad para unificar la dispersión y, por otra parte, a explicar la distintividad ante la sociedad no mapuche (Briones 2006).

A partir del año 2001, comenzó a aparecer en el movimiento mapuche un nuevo planteo marcado por diacríticos de edad. El planteo de estos (nuevos) "jóvenes" introdujo un horizonte heterogéneo de discursos y prácticas en la arena política, estableciendo continuidades, redefiniciones y rupturas con respecto a la generación anterior de activistas. Una de las particularidades del activismo de estos "jóvenes mapuche" que más impacto público tuvo fue la vinculación que esta nueva generación estableció entre su experiencia como jóvenes de la periferia urbana y su pertenencia al Pueblo Mapuche.

Si los "jóvenes" de 1990 ponían el énfasis en la recuperación cultural, los "jóvenes" del 2001 partieron de allí para visibilizar la heterogeneidad de las trayectorias mapuche históricas y presentes (Kropff 2004). Tenemos, entonces, un horizonte discursivo que incluye la "recuperación cultural" y la heterogeneidad de las trayectorias mapuche, incorporando experiencias ruralizadas y urbanizadas. En la comunidad Newen Ñuke Mapu, los procesos de memoria recreados por los jóvenes están atravesados por los lugares sociales que el activismo mapuche de 1990 construyó en el ámbito público, por las trayectorias heterogéneas que se visibilizaron a partir de 2001, y también por los lugares que fueron habilitados en el ámbito judicial.

En la provincia de Río Negro, el proceso de disputa de aboriginalidad en términos de derechos con efectos en el ámbito judicial comenzó en la década de 1980. El hito principal fue la lucha por la Ley Integral del Indígena 
cuya sanción se logró en 1987 implicando un importante proceso de organización indígena y campesina. La organización de referencia en ese proceso de demanda fue el Consejo Asesor Indígena (CAI) que también acompañó demandas territoriales en la década de 1990 (Cañuqueo et al. 2005). La estrategia de reafirmación territorial del lof Mariano Epulef se inició en el marco de reivindicación que llevaban adelante otras comunidades mapuche de la Línea Sur en coordinación con el CAI.

Las voces públicas del lof en este proceso son de miembros que, en algunos casos, tienen experiencia de participación en agrupamientos de jóvenes mapuche que emergieron en la primera década del siglo XXI. Eran niños cuando acompañaban a sus padres y tíos a las asambleas del CAI en los ochenta. Viven en Anecón Chico y en Bariloche. Su posicionamiento público tiene que producir sentido en torno a una doble subalternidad: en términos de aboriginalidad y en términos de edad. En algunos casos se suma el género, produciendo una subalternidad triple. La arena de disputa política y jurídica implica una necesaria reflexión sobre la cuestión de la aboriginalidad y la condición comunitaria: deben probar su "autenticidad". Este proceso incluye también contextos familiares en los cuales la posición etaria produce efectos específicos.

\section{Genealogías entramadas}

Durante el trabajo de campo previo a la conformación de la Newen Nuke Mapu, participé de una conversación nocturna en la casa de Anecón Chico donde nos alojaban. Quizás valga la pena aclarar aquí que el idioma que se habla en el presente en las zonas rurales es el castellano, aunque se incluyen eventualmente algunos términos en mapuzugun. Sin embargo, aunque los jóvenes son monolingües en castellano, la generación anterior incluye personas bilingües y, según ellos cuentan, la generación de los abuelos hablaba preponderantemente en mapuzugun en la vida cotidiana.

Esa noche, los/as jóvenes de la familia empezaron a reflexionar sobre los roles de las diferentes personas en el lof Mariano Epulef. En ese diálogo empezaron a incluir referencias a los roles comunitarios y ceremoniales tradicionales que son reivindicados públicamente desde que se visibilizaron en la década de 1990 en el marco de la "recuperación cultural". Lo que empezó siendo un juego acabó operando como un lenguaje útil para dar cuenta de la estructura al interior del lof. A la luz de los roles estipulados por ese horizonte discursivo, los/as jóvenes produjeron sentido sobre su propio proceso comunitario y sus relaciones familiares. Así, asignaron a uno de sus primos el rol de "sargento pita wigka", figura fundamental del kamarikun, "por la 
mística", dijo una de las jóvenes: "no habla, sólo mira y todos lo siguen". Una de las tías con mayor carácter fue definida como "pijankuse", uno de los roles femeninos ceremoniales más importantes; otra como "tayvlufe", que colabora con la pijankuse. El tío que vivía en la casa era "Capitán pita wigka" porque tenía un apellido distinto al del "sargento" y su familia había llegado después (en referencia al proceso de conformación comunitaria). Pero también porque llevaba a los/as niños/as en su camioneta para todos lados cuando iba a visitar a los vecinos o al pueblo. "De él aprendimos a manejar las relaciones a nivel local", dijo alguien. Además, dijeron que ese tío era un poco "vlmenche" porque sabía administrar bien los "negocios" del campo. Otro tío, que había participado activamente en el CAI en los ochenta fue denominado en castellano como "jefe de relaciones exteriores", porque su experiencia habilitó la articulación con las organizaciones supra-comunitarias y también fue ejemplo para los jóvenes que en el presente interactuaban con ellas y con agencias gubernamentales.

En esa conversación se destacó que las mujeres del lof permitieron la continuidad comunitaria porque fueron "adoptando" niños. Adoptar, en ese marco, tenía que ver con mantener a los/as hijos/as y los/as sobrinos/as en el lof evitando la dispersión. Algunas tomaron a ciertos sobrinos/as en particular para formarlos, al estilo antiguo, como chokun [hijo de crianza]. Las que migraron a la ciudad "entendieron la mística", dijo una joven, porque permitieron que sus hijos/as siguieran yendo al campo y no perdieran ese vínculo, aplicando una noción práctica de territorialidad basada en la ocupación a través del tránsito. Los/as jóvenes fueron clasificados como seguidores/as de uno/a u otro/a de la generación anterior, de acuerdo a los roles que ocupan en el proceso de reafirmación territorial del presente: los/as que manejan las relaciones a nivel local, los/as que hablan con los abogados y los jueces, los/as que participan activamente en el CAI, los/as que manejan los "negocios", los/as que mantienen la "mística". "El problema está cuando uno quiere ocupar un rol que no le corresponde", dijo uno de los jóvenes.

Las distintas tradiciones (familiares, organizacionales, mapuche, no mapuche, estatales) puestas en juego en el caso de la comunidad Newen Nuke Mapu y, específicamente, del lof Mariano Epulef, producen un entramado de interpelaciones, articulaciones de agencia y subjetivaciones con diferente grado de sedimentación en el que los jóvenes ponen en juego y redefinen su posicionamiento de edad para legitimar su voz haciendo un uso creativo de sus competencias culturales.

Cité más arriba ejemplos en los que se produce un efecto de transmisión intergeneracional de prácticas a través de diferentes mandatos. En el primer ejemplo, la joven transita los pasos ceremoniales de su abuela en el 
kamarikun; en el segundo, el joven lleva adelante el consejo de la tía en la reafirmación territorial. En ambos casos hay otras personas presentes en los gestos individuales de cada uno: son gestos comunalizadores que actúan tradiciones heredadas, resignificadas en el contexto presente. En la conversación familiar de la que participé también se produjo sentido en torno a los roles comunitarios, apelando heurísticamente a categorías que provienen de contextos ceremoniales y que fueron activadas en el proceso de organización de la década de 1990, para promover el proyecto comunalizador del Pueblo Nación Mapuche. Además de estos contextos ceremoniales, familiares, comunitarios, los jóvenes interactúan con agencias estatales y con medios de comunicación, en función de legitimar el proceso de demanda. Una de las agencias fundamentales es el poder judicial que acaba teniendo efectos sobre el discurso público de los jóvenes.

En los expedientes judiciales, el lof Mariano Epulef debe narrar su historia en función de legitimar su demanda. Los documentos son elaborados por los jóvenes en diálogo con los abogados y activistas del CAI. Fruto de ese diálogo, el registro discursivo queda hegemonizado por señalamientos que tienen que ver con el lenguaje judicial, pero entramados con otros registros. ${ }^{5}$ Así, las referencias al territorio disputado, deben marcarse en términos de "legua", "lote", "sección", "departamento", "deslinde", "amojonamiento", "mensura", etc., y sus prácticas de ocupación se definen en términos de "situación dominial", "mejoras", etc. Sin embargo, los documentos están también atravesados por el registro discursivo del movimiento mapuche, incluyendo términos en mapuzugun - como lof, lamgen [persona mapuche ${ }^{6}$, GuluMapu [Territorio Mapuche al oeste de los andes], PuelMapu [Territorio Mapuche al este de los andes] - y términos en castellano instalados por el movimiento político - como "Territorio Mapuche" y "Pueblo" (con mayúscula).

La participación activa de los jóvenes en la elaboración de esos textos implica un uso creativo del capital simbólico acumulado en sus trayectorias rurales, urbanas, escolares, universitarias, activistas. Además de su conocimiento del discurso judicial y de las organizaciones mapuche, la experiencia activista de los jóvenes incluye la participación en radios comunitarias y la edición de publicaciones destinadas a circuitos juveniles (Cañuqueo y Kropff 2007). Esto redunda en un uso activo de estos medios en el proceso de demanda. Como consecuencia, la agencia de los jóvenes se despliega en ámbitos activistas, judiciales, mediáticos, comunitarios y familiares, desplegando y combinando creativamente elementos de una serie de registros diferentes que incluyen los géneros discursivos mapuche (Cañuqueo, 2004).

El capital simbólico puesto en juego se basa en experiencias históricas generacionalmente específicas, que tienen que ver con el momento en el 
que los jóvenes ingresaron como actores en la arena política y con el posicionamiento en una determinada genealogía familiar y comunitaria. Otras generaciones de la comunidad cuentan con otros capitales y su movilidad está estructurada de manera distinta. El contraste entre los valores morales y éticos fundados en experiencias históricas diferentes de relación con el estado, los latifundistas, las organizaciones y el sistema judicial produce lógicas diferentes que se ponen de manifiesto en el diálogo intergeneracional al interior de las comunidades. Este diálogo no siempre redunda en resultados armónicos. En el caso del lof Mariano Epulef, el proceso formalizado de paso de la toma de decisiones a las generaciones genealógicas jóvenes permite el despliegue de su lógica generacional. En otros casos, son otras las lógicas que acaban operando.

\section{Palabras finales}

En una de nuestras visitas, los jóvenes del lof Mariano Epulef nos llevaron a hacer un recorrido por las tierras disputadas. Ese recorrido tuvo como paradas las diferentes taperas que marcan la presencia antigua de pobladores que, en su mayoría, fueron desalojados por las presiones del usurpador en connivencia con los poderes del estado. Las marcas de este desplazamiento histórico eran arboledas de álamos y sauces y restos de corrales de piedra y de viviendas. Para llegar a las taperas, usamos los caminos vecinales que marcan la ocupación cotidiana de esos espacios a través de la circulación. Los caminos, a su vez, estaban atravesados por alambrados. Algunos de esos alambrados marcaban el uso de los cuadros por parte del lof y otros eran los trazos de la usurpación. Los alambres "caminan de noche", dicen los mayores, refiriéndose a la práctica recurrente de los latifundistas de alambrar cuando los pobladores no están atentos.

Recorrimos las tierras que en los expedientes figuran como "legua $\mathrm{n}^{\mathrm{o}}$ $\mathrm{x}$ " del "lote $\mathrm{n}^{\mathrm{o}} \mathrm{x}$ " de la "sección $\mathrm{x}$ " que, en el recorrido, se volvían lugares de memoria. Los hechos de la historia comunitaria aparecían espacializados en relaciones de antigua vecindad (vecinos que habitaron esos lugares y vecinos que colaboraron en la construcción de corrales y casas), memorias de la usurpación, de desalojos violentos, pero también de riqueza material expresada en el tamaño de casas y corrales. Los hechos de la historia de la genealogía familiar también se encontraban en esos mojones de árboles y piedras: muertes y nacimientos, matrimonios y separaciones, etc. Finalmente, también la biografía de los jóvenes se expresaba en esos espacios: los lugares de juego y aventuras de la infancia, los lugares permitidos y prohibidos, los 
lugares cargados de fuerzas espirituales: aguadas, cerros, arboledas, piedras.

El proceso de recuperación y reafirmación territorial mapuche en áreas rurales se incluye dentro de una reflexión mayor que articula trayectorias individuales y colectivas. Si la generación anterior de activistas logró instalar una definición geopolítica de Territorio en la que se destaca la pre-existencia a los estados nacionales y se fundamenta la recuperación territorial, los jóvenes del siglo XXI colocan el énfasis en las trayectorias de desplazamiento que construyen territorialidad más allá de la dicotomía entre lo rural y lo urbano, basada en nociones estáticas de Territorio.

"¿Y costó mucho convencer a la familia de hacer eso, de recuperar?", le pregunté a uno de los jóvenes que nos llevó en el recorrido por las taperas del territorio reafirmado. Me contestó:

No costó porque fue por la memoria, la pérdida y el deseo de tenerlo siempre. Ellos hablaban de que algún día iban a voltear el alambre. (...) y siempre andábamos por ahí. O sea nunca... yo no recuerdo que nos hubiesen sacado cuando éramos chicos. Yo andaba mucho en esos campos. Íbamos a buscar piches, a andar de a caballo.

En esa respuesta está presente la memoria de pérdida y el deseo de los mayores y la memoria de la cotidianidad de la infancia de los jóvenes. El Territorio contiene marcas que espacializan esas dos experiencias y, en ese sentido, se vuelve lenguaje para un diálogo intergeneracional. De este modo, la genealogía comunitaria opera transmitiendo derechos y obligaciones con respecto a ese espacio/lugar. Los hijos, nietos, sobrinos de quienes firmaron la carta de 1941 se constituyen en herederos de la demanda del derecho al territorio y, de ese modo, la comunidad Newen Ñuke Mapu se configura en torno a esa reivindicación. El recorrido por la experiencia de los jóvenes del lof Mariano Epulef refleja sólo una de las genealogías trazadas y retomadas en la reunión.

Entonces, volviendo a las preguntas iniciales, la apuesta en este artículo fue abordar el análisis de la edad como clivaje en un contexto etnográfico en el que se encuentra entramada con el clivaje étnico. El proceso de comunalización de la Newen Ñuke Mapu construye como tópico, específicamente, la etnicidad al plantearse la configuración de una comunidad mapuche. En ese sentido, la disputa en términos de aboriginalidad supone visibilizar la presencia indígena en un área en la que fue invisibilizada en términos de colectivo social. A pesar de las demandas colectivas que constan en los archivos gubernamentales, de las que la carta mencionada en la reunión es sólo un ejemplo, el estado nunca reconoció la presencia de comunida- 
des indígenas en esos parajes. Sin embargo, a pesar de la invisibilización, la trama de relacionalidad que reúne a los pobladores mapuche, disputó los términos de su presencia de diferentes modos a lo largo de la historia. Inscritas en esta trayectoria, las demandas del presente necesitan incluir argumentaciones que demuestren autenticidad en términos de identidad étnica y de condición comunitaria. Podemos decir que el clivaje etario opera, en este proceso, suturando experiencias históricas con deberes de parentesco, a través del anclaje generacional tanto en términos políticos como genealógicos con el efecto de primordializar tanto la aboriginalidad como la noción de comunidad y de inscribirlas en el espacio devenido Territorio.

Recebido em 14 de abril de 2015

Aprovado em 10 de junho de 2016

Laura Kropff. Instituto de Investigaciones en Diversidad Cultural y Procesos de Cambio (IIDyPCa). CONICET. Universidad Nacional de Rio Negro, Bariloche/ Río Negro, Argentina. E-mail: <laukropff@gmail.com>

\section{Notas}

1 Los datos del censo 2010 no fueron tomados con los mismos criterios y, al no haberse realizado una encuesta complementaria, no se puede contar con datos tan específicos como los del censo 2001. En 2010, el criterio fue aplicar la pregunta por la auto identificación indígena sólo en un porcentaje de cédulas censales, no en la totalidad. A partir de esa técnica se llegó a los siguientes resultados: del total de 955.032 personas autorreconocidas como indígenas en todo el país, 205.009 se identificaron como mapuche, es decir el 21,5\%.

2 La carta forma parte del Expediente del Archivo Histórico Provincial de Río Negro $n^{\circ} 140074 / 41$, que fuera hallado a instancias de la reivindicación territorial realizada por el lof Mariano Epulef, integrante de la comunidad.

3 Cabe destacar que la consanguinidad y el apellido constituyen elementos clave para legitimar el derecho a la tierra según la legislación vigente que pauta la herencia de la propiedad. Sin embargo, existen en la comunidad formas tradicionales de familia que incluyen la figura del hijo de crianza que no lleva el apellido del padre ni tiene vínculos de consanguinidad. Identificamos genealogías basadas en esta figura que entran en disputa con las que incluyen la apelación a la consanguinidad. 
4 Mientras las trayectorias urbanas ponen en cuestión la ruralización de la aboriginalidad para incluirse dentro de la experiencia colectiva del Pueblo Mapuche, tienden a refrendar territorializaciones etarias hegemónicas que circunscriben lo joven a los espacios urbanos. Por otro lado, las trayectorias rurales de los jóvenes mapuche desafían la territorialización hegemónica de la juventud, habilitando y disputando la legitimidad de su presencia en el campo. La confluencia de estas trayectorias acaba concibiendo una subjetividad juvenil mapuche que transita a través de lo que se denomina como "cultura" y, en la misma operación, se des-ruraliza y se des-urbaniza (ver Kropff 2011).

5 Los fragmentos de documentos que pertenecen a expedientes judiciales en curso no se pueden reproducir aquí por razones legales.

6 Si el término lamgen es usado por un hombre, refiere a una mujer mapuche, mientras que si es usado por una mujer puede referir tanto a hombres como a mujeres. El hecho de que en los documentos del lof se encuentre el término lamgen y no el término peñi (que es el término usado por los hombres para referirse a otros hombres mapuche) pone en evidencia un posicionamiento autoral femenino así como una referencia explícita a la pluralidad genérica de los referentes del discurso escrito. Cuando la posición de enunciación es masculina, la fórmula recurrente para reconocer la pluralidad de género es "peñi y lamgen".

\section{Bibliografía}

ALONSO, Ana. 1994. "The politics of space, time and substance: State formation, nationalism, and ethnicity". Annual Review of Anthropology, 23:379-405.

BARTH, Frederik. 1976 [1969]. "Introducción". In: Los grupos étnicos y sus fronteras. La organización social de las diferencias culturales. México: Fondo de Cultura Económica. pp. 7-49.

BECKETT, Jeremy (ed.). 1988. Past and present. The construction of aboriginality. Canberra: Aboriginal Studies Press.

BRIONES, Claudia. 1998. La alteridad del "Cuarto Mundo". Una deconstrucción antropológica de la diferencia. Buenos Aires: Ediciones del Sol.
2006. "Questioning state geographies of inclusion in Argentina: the cultural politics of organizations with Mapuche leadership and philosophy". In: Cultural agency in the Americas. Durham: Duke University Press. pp. 248-278.

; DELRIO, Walter. 2002. "Patria sí, colonias también. Estrategias diferenciales de radicación de indígenas en Pampa y Patagonia (1885-1900)". In: A. Teruel, M. Lacarrieu y O. Jerez (comps.), Fronteras, ciudades y estados. Tomo I. Córdoba: Alción Editora. pp. 45-78.

.; SIFFREDI, Alejandra. 1989. “Discusión introductoria sobre los límites teóricos de lo étnico". Cuadernos de Antropología, 3:5-24. 
BROW, James. 1990. "Notes on community, hegemony, and uses of the past". Anthropological Quarter, 63(1):1-6. Traducción de la cátedra de etnolingüística de la Facultad de Filosofía y Letras, U.B.A. Mimeo.

CAÑUQUEO, Lorena. 2004. “El Territorio Mapuche desde la perspectiva del ngutram". Asuntos Indígenas, 4(4): 33-37.

. 2010. "Discursos sobre pertenencias mapuche: las formas habilitadas de pensar la 'comunidad mapuche' en río Negro". Tesis de Licenciatura en Comunicación Social. Facultad de Derecho y Ciencias Sociales, Universidad Nacional del Comahue. . 2011 "'Tramitar' la comunidad indígena en Río Negro. Activismo, co-gestión y políticas de reconocimiento". Actas del X Congreso Argentino de Antropología Social. Buenos Aires: Editorial de la Facultad de Filosofía y Letras, Universidad de Buenos Aires, Mimeo.

.; KROPFF, Laura. 2007. "MapUrbe'zine: los cuerpos de 'la lucha' en el circuito Heavy-Punk Mapuche". E-misférica, 4(2). Publicación electrónica: http://hemisphericinstitute. org/journal/4.2/eng/en42_pg_canuqueo_kropff.html

; KROPFF, Laura; RODRÍGUEZ, Mariela; VIVALDI, Ana. 2005. "Tierras, indios y zonas en la provincia de Río Negro". In: C. Briones (comp.), Cartografías argentinas. Políticas indigenistas y formaciones provinciales de alteridad. Buenos Aires: Editorial Antropofagia. pp. 111-139.

.; KROPFF, Laura; PÉREZ, Pilar. 2007. “El 'paraje' y la 'comunidad' en la construcción de pertenencias colectivas mapuche en la provincia de Río Negro". VIII Congreso Argentino de Antropología Social, Universidad Nacional de Salta, 19 al
22 de septiembre de 2006. Actas en CD, Salta: EDUNSA.

.; KROPFF, Laura; PÉREZ, Pilar 2008. "¿Un "ulmenche" en el territorio patagónico del siglo XX?: el caso de Mariano Epulef". En 3ras Jornadas de Historia de la Patagonia. San Carlos de Bariloche, 6 al 8 de noviembre, Universidad Nacional del Comahue. Mimeo.

.; KROPFF, Laura; PÉREZ, Pilar. 2015. "A la sombra del Estado: comunalización indígena en parajes de la pre cordillera de Río Negro, Argentina" Revista del Museo de Antropología, 8 (2): 159-170.

CAÑUMIL, Pablo; RAMOS, Ana. 2012. "Algunas reflexiones sobre los procesos de formación del lof". Ponencia presentada en Coloquio Internacional "Prácticas de pertenencia trans-estatales y trans-categoriales", 9 y 10 de abril, IIDyPCA, CONICET-UNRN, Sociedad Suiza de Americanistas. Bariloche. Mimeo.

CARDOSO DE OLIVEIRA, Roberto. 1971. "Identidad étnica. Identificación y manipulación". América Indígena, XXX(4): 923-953.

CHAVES, Mariana. 2010. Jóvenes, territorios y complicidades. Buenos Aires: Espacio.

COORDINACION DE ORGANIZACIONES MAPUCHE TAIÑ KIÑE GETUAM -PARA VOLVER A SER UNO-. 1995. "Autonomía y pueblos originarios". Primer Seminario Regional El Derecho Internacional y los Pueblos Originarios. Neuquén, 29 de Septiembre al 2 de Octubre, Universidad Nacional del Comahue. Mimeo.

DELRIO, Walter. 2005. Memorias de expropiación. Sometimiento e incorporación indígena en la Patagonia 1872-1943. Bernal: Universidad Nacional de Quilmes. 
DURHAM, Deborah. 2000. "Youth and the social imagination in Africa: introduction to parts 1 and 2". Anthropological Quarterly, 73(3):113-120.

ESCOLAR, Diego. 2008. "El repartimiento de prisioneros indígenas en Mendoza durante y después de la Campaña del Desierto". III Jornadas de Historia de la Patagonia, Universidad Nacional del Comahue, San Carlos de Bariloche, 6 al 8 de noviembre. Mimeo.

EVANS-PRITCHARD, Edward E. 1987 [1940]. Los Nuer. Barcelona: Anagrama.

GONZALEZ CANGAS, Yanko. 2003. "Juventud rural. Trayectorias teóricas y dilemas identitarios". Revista Nueva Antropología, XIX(63):153-175.

GUPTA, Akhil; FERGUSON, James. 1992 "Beyond 'culture': space, identity and the politics of difference". Cultural Anthropology, 7(1): 6-23.

IRVINE, Judith T. 1978 "When is genealogy history? Wolof genealogies in comparative perspective". American Ethnologist, 5(4): 651-674.

KERTZER, David I. 1978 "Review: theoretical developments in the study of age-group systems". American Ethnologist, 5(2): 368-374.

KROPFF, Laura. 2002 "Indios, chilotes y vecinos en una ciudad patagónica". Cuadernos de antropología social, Dossier $\mathrm{n}^{\circ} 16$ (Conflictos interétnicos en la sociedad contemporánea): 211-229.

. 2004. "Mapurbe: jóvenes mapuche urbanos". KAIROS-Revista de Temas Sociales, 14. Universidad de San Luis, Publicación electrónica: http://www2.fices.unsl.edu.ar/ kairos/k14-05.htm

. 2011 "Los jóvenes mapuche en Argentina: entre el circuito punk y las recuperaciones de tierras". Alteridades, 21(42):77-89.
; STELLA, Valentina. 2010. "Juventud y etnicidad en Latinoamérica: un estado del arte". Coloquio Internacional juventud, etnicidad, ruralidad y movimientos translocales en Latinoamérica, Instituto de Investigaciones en Diversidad Cultural y Procesos de Cambio. Sede Andina, Universidad Nacional de Río Negro. Bariloche, 21 y 22 de octubre. Mimeo.

LENTON, Diana. 2005. "De centauros a protegidos. La construcción del sujeto de la política indigenista Argentina desde los debates parlamentarios (1880-1970)". Tesis Doctoral en Antropología, Facultad de Filosofía y Letras, Universidad de Buenos Aires. LEWKOWICZ, Ignacio. 2003. "Subjetivación post-estatal \# 5 Generaciones y constitución política", desgrabación de la reunión del Grupo Viernes del 09-05-03 integrado por Raquel Bozzolo, Elena De la Aldea, Pancho Ferrara, Mirta Groshaus, Raquel Jaduszliwer, Marta L'Hoste, Beatriz López, Sol Pelaez, Nina Stein y coordinado por Ignacio Lewkowicz. Recuperado el 2 de septiembre de 2008 de: www.estudiolwz.com.ar/ protoWeb/lwz03/smn/Viern/ViernesGenPolWeb.pdf

MASES, Enrique. 2002. Estado y cuestión indígena. El destino final de los indios sometidos en el sur del territorio (1878-1910). Buenos Aires: Prometeo.

MAYBURY-LEWIS, David. 1974 [1967]. Akwè-Shavante society. New York, London, Toronto: Oxford University Press.

MÜLLER-DEMPF, Harald K. 1991 "Generation-sets: stability and change, with special reference to Toposa and Turkana societies". Bulletin of the School of Oriental and African Studies, 54(3):554-567. 


\section{ORGANIZACIÓN MAPUCHE NEWEN-} TUAYIÑ Y PU WECHE FISKE MENUKO (Jóvenes Mapuche de Fiske Menuco). 2001. "Postura Mapuche frente a la incorporación de "la variable indígena" en el Censo Nacional de Población y Vivienda 2001". Recuperado el 7 de agosto de 2005 de: http://www.mapuche.info/mapuint/ Newentuayin011000.html

PACHECO DE OLIVEIRA, João. 1998 "Uma etnologia dos 'índios misturados'? Situação colonial, territorialização e fluxos culturais". Mana. Estudos de Antropologia Social, 4(1):47-77.

PÉREZ, Pilar. 2009. "Las policías fronterizas: mecanismos de control y espacialización en los territorios nacionales del sur a principios del siglo XX". XII Jornadas Interescuelas-Departamentos de Historia, Universidad Nacional del Comahue, San Carlos de Bariloche, 28 al 31 de octubre. Mimeo.

PÉREZ RUIZ, Maya Lorena. 2008 "Presentación: jóvenes indígenas en América Latina: żglobalizarse o morir?". In: Jóvenes indígenas y globalización en América Latina. México: INAH. pp. 9-41.

RADCLIFFE-BROWN, Alfred Reginald. 1929 "13. Age organization-terminology". Man, 29:21.

RADOVICH, Juan Carlos; BALAZOTE, Alejandro. 1995. "Transiciones y fronteras agropecuarias en Norpatagonia". In: H. Trinchero (ed.), Producción doméstica y capital. Estudios desde la antropología económica. Buenos Aires: Biblos. pp. 63-80.

. 2000. "Mapuches en Neuquén: conflictos en el orden económico y simbólico". In: El resignificado del desarrollo. Buenos Aires: UNIDA. pp. 257-271.

RAMOS, Ana; DELRIO, Walter. 2005. "Trayectorias de oposición. Los mapuches y tehuelches frente a la hegemonía en Chubut". In: C. Briones (comp.), Cartografías argentinas: políticas indígenas y formaciones provinciales de alteridad. Buenos Aires: Antropofagia. pp.79-118.

SILVERSTEIN, Michael. 1976. "Shifters, linguistic categories, and cultural description". In: K. Basso; H. Selby (eds.), Meaning in anthropology. Albuquerque: University of New Mexico. pp. 11-55.

SVAMPA, Maristella. 1994. El dilema argentino: civilización o barbarie. De Sarmiento al revisionismo peronista. Buenos Aires: El Cielo por Asalto.

SZULC, Andrea. 2004. "'Mapuche se es también en la waria (ciudad)'. Disputas en torno a lo rural, lo urbano y lo indígena en la Argentina". Política y Sociedad, 41(3):167-180.

WILLIAMS, Raymond. 2001 [1973]. El campo y la ciudad. Buenos Aires: Paidos. 
ENTRE GENEALOGÍAS FAM ILIARES Y GENEALOGÍAS POLÍTICAS: JÓVENES EN UN PROCESO DE COMUNALIZACIÓN MAPUCHE EN ARGENTINA

\section{Resumo}

A partir de uma perspectiva teórica que entende que a idade e a etnia são clivagens estreitamente interligadas, este artigo discute o seu impacto sobre o processo de comunalizaçao da comunidade mapuche Newen Nuke Mapu (Rio Negro, Argentina) e, especificamente, o caso do Lof Mariano Epulef incluído nessa comunidade. Nesse contexto, o objeto de análise é a maneira pela qual as articulações contemporâneas da geração de "jovens mapuche" produzem significações, por um lado, sobre a genealogia política do movimento mapuche que se desenvolveu nas últimas décadas do século XX e, por outro, sobre as genealogias das famílias e da comunidade. Ao mesmo tempo, o artigo aproxima-se do modo pelo qual essas genealogias definem as articulações das gerações. Esta tarefa inclui estudar a apropriação crítica dos discursos políticos mapuche por "jovens" nos processos das comunidades rurais.

Palavras-chave: Etnia, Gerações, Política, Mapuche.

ENTRE GENEALOGIAS FAM ILIARES E GENEALOGIAS POLÍTICAS: JOVENS EM UM PROCESSO DE COMUNALIZAÇÃO MAPUCHE NA ARGENTINA

\section{Resumen}

A partir de una perspectiva teórica que entiende que edad y etnicidad constituyen clivajes complejamente entramados, en este artículo se aborda su incidencia en el proceso de comunalización de la comunidad mapuche Newen Nuke Mapu (Río Negro, Argentina) y, específicamente, en el caso del Lof Mariano Epulef que la integra. En ese contexto se analizan los modos en que la articulación generacional de los "jóvenes mapuche" en el presente produce sentido sobre -y es definida por- tanto la genealogía política del movimiento de fines del siglo XX como la genealogía familiar y comunitaria. Esto implica analizar la reelaboración política generacional que los "jóvenes" realizan de los discursos organizacionales mapuche a partir de su puesta en práctica en el proceso de articulación comunitaria rural.

Palabras clave: etnicidad; generaciones; política; mapuche.

\section{BETWEEN FAMILY GENEALOGIES}

AND POLITICAL GENEALOGIES: YOUNG PEOPLE IN A MAPUCHE COMMUNALISATION PROCESS IN ARGENTINA

\section{Abstract}

Drawing on a theoretical perspective that understands age and ethnicity as intertwined cleavages, this article discusses their effects on the communalisation process of the Mapuche community of Newen Nuke Mapu (Rio Negro, Argentina). It focuses specifically on the case of Lof Mariano Epulef in the context of this community. The analysis deals with how contemporary generational articulations of "young Mapuche" give meaning to the political genealogy of the movement that developed in the last decades of the twentieth century and also, at the same 
time, to family and community genealogies. The article likewise addresses the ways in which these genealogies define generational articulations. This task involves studying the critical generatio- nal appropriation of political Mapuche discourses by "young Mapuche" within rural communalisation processes.

Keywords: ethnicity; generations; politics; mapuche. 
